\title{
The $\alpha$-Subunit of the Chloroplast ATP Synthase of Tomato Reinforces Resistance to Gray Mold and Broad-Spectrum Resistance in Transgenic Tobacco
}

\author{
Chao Gong, ${ }^{1,2}$ Mo-zhen Cheng, ${ }^{1}$ Jing-fu Li, ${ }^{3}$ Hong-yu Chen, ${ }^{1}$ Zhen-zhu Zhang, ${ }^{1,4}$ Hao-nan Qi, ${ }^{1}$ Yao Zhang, ${ }^{1}$ Jiayin Liu, ${ }^{3}$ \\ Xiu-ling Chen, ${ }^{3, \dagger}$ and Ao-xue Wang $1,3, \dagger$ \\ ${ }^{1}$ College of Life Sciences, Northeast Agricultural University, Harbin 150030, People's Republic of China \\ ${ }^{2}$ Guangdong Key Laboratory for New Technology Research of Vegetables, Vegetable Research Institute, Guangdong Academy of Agricultural \\ Sciences, Guangzhou 510640, People's Republic of China \\ ${ }^{3}$ College of Horticulture and Landscape Architecture, Northeast Agricultural University, Harbin 150030, People's Republic of China \\ ${ }^{4}$ College of Life Sciences, Agriculture and Forestry, Qiqihar University, Qiqihar 161006, People's Republic of China \\ Accepted for publication 5 August 2020.
}

ABSTRACT

\begin{abstract}
Chloroplast ATP synthase (cpATPase) is responsible for ATP production during photosynthesis. Our previous studies showed that the cpATPase CF1 $\alpha$ subunit (AtpA) is a key protein involved in Clonostachys rosea-induced resistance to the fungus Botrytis cinerea in tomato. Here, we show that expression of the tomato atpA gene was upregulated by $B$. cinerea and Clonostachys rosea. The tomato atpA gene was then isolated, and transgenic tobacco lines were obtained. Compared with untransformed plants, atpA-overexpressing tobacco showed increased resistance to $B$. cinerea, characterized by reduced disease incidence, defense-associated hypersensitive response-like reactions, balanced reactive oxygen species, alleviated damage to the chloroplast ultrastructure of leaf cells, elevated levels of ATP content and cpATPase activity, and enhanced expression of genes related to
\end{abstract}

carbon metabolism, photosynthesis, and defense. Incremental $\mathrm{Ca}^{2+}$ efflux and steady $\mathrm{H}^{+}$efflux were observed in transgenic tobacco after inoculation with $B$. cinerea. In addition, overexpression of atp $A$ conferred enhanced tolerance to salinity and resistance to the fungus Cladosporium fulvum. Thus, AtpA is a key regulator that links signaling to cellular redox homeostasis, ATP biosynthesis, and gene expression of resistance traits to modulate immunity to pathogen infection and provides broad-spectrum resistance in plants in the process.

Keywords: $\alpha$ subunit of cpATPase, abiotic disorders, ATP, B. cinerea resistance, biotechnology, broad-spectrum resistance, disease resistance, molecular, plant immune responses, plant stress, tomato
Gray mold of tomato (Solanum lycopersicum) is a devastating disease caused by the necrotrophic fungal pathogen Botrytis cinerea. Previous studies suggest that $B$. cinerea is not restrained by host-mediated localized hypersensitive responses (HRs), but rather by necrotic tissues (Patel et al. 2015). However, Audenaert et al. (2002) showed that the functional deletion of abscisic acid empowered sitiens tomato mutants and increased resistance to $B$. cinerea via a quick epidermal $\mathrm{HR}$ and associated cell wall fortifications derived from the phenylpropanoid pathway. Resistance to $B$. cinerea, as with most necrotrophs, depends on the balance between cell death and survival (Mengiste 2012). Yet the effects of HR-mediated defense tactics in plants against $B$. cinerea remain unknown (Seifi et al. 2013).

${ }^{\dagger}$ Corresponding authors: A. Wang; axwang@neau.edu.cn, and

X. Chen; chenx@neau.edu.cn

Funding: This work was financially supported by grants from the National Natural Science Foundation of China (31872120), National Key R\&D Program of China (2017YFE0105000), National High Level Talents Special Support Program (W03020273 to A. Wang), National Science Foundation of China (31801875 to C. Gong), and Academic Backbone Project of Northeast Agricultural University (17XG06 to X. Chen).

C. Gong and M. Cheng contributed equally to this work.

*The $e$-Xtra logo stands for "electronic extra" and indicates that supplementary figures and eight supplementary tables are published online.

The author(s) declare no conflict of interest.

(c) 2021 The American Phytopathological Society
Because of the lack of tomato cultivars with resistance against gray mold, no such varieties have yet been bred. Defense priming can be induced by phytopathogens and nonpathogenic beneficial organisms, among others, some of which can elicit the gene expression of defensive traits (Conrath et al. 2015; MartinezMedina et al. 2016; Verhagen et al. 2009). Clonostachys rosea is an effective antagonistic fungus to $B$. cinerea. In our previous studies, both the disease incidence and the disease index were significantly reduced in Clonostachys rosea-inoculated tomato compared with $B$. cinerea inoculation alone by the regulation of phytohormone contents, secondary messengers, and defense-related enzymes activities in tomato; notably, the AtpA was identified as the abundant protein in tomato treated with Clonostachys rosea (Gong et al. 2017; Mouekouba et al. 2014). Those results indicated that the enhanced defense of tomato to $B$. cinerea infection was primed by Clonostachys rosea with AtpA perhaps involved in B. cinerea resistance. However, the mechanisms by which AtpA might suppress $B$. cinerea and influence subsequent host-defense response patterns have yet to be comprehensively explored.

Plants have evolved a sophisticated innate immune system that can be activated when they are attacked by one or more pathogens. Generally, this plant innate immune system can be summarized in a four-phase "zigzag" model (Jones and Dangl 2006). In phase 1, plants' first line of defense relies on recognizing pathogenassociated molecular patterns (PAMPs) by specific extracellular surface pattern-recognition receptors, which leads to PAMP-triggered immunity (PTI) that limits pathogen colonization of the host (Dangl et al. 2013). In phase 2, successful pathogens can suppress the host's PTI response via the production of effectors, which leads to the onset of disease, and this results in effector-triggered 
susceptibility. In phase 3, plants in turn evolve a group of specific disease resistance genes ( $R$ genes), some of which can specifically recognize a given effector, and this triggers a second-tier immune response called effector-triggered immunity (ETI) (Xu et al. 2019). In phase 4, pathogens are able to suppress ETI by shedding or diversifying the recognized effector gene or by acquiring new effectors. Natural selection drives new plant $R$ genes to specially recognize one of the newly acquired effectors so that ETI can be triggered again in the host (Jones and Dangl 2006).

Many effectors can suppress one or more components of PTI and ETI responses and these contribute to the enhancement of pathogen virulence (Jones and Dangl 2006; Xu et al. 2019). For example, haustorium-specific protein Pst_12806 was identified as a Puccinia striiformis f. sp. tritici effector that interacted with TaISP, a putative component of the cytochrome b6-f complex, and attenuates the rate of photosynthesis and inhibits plant defenses (Xu et al. 2019). Effector Pst18363 was shown to target Nudix hydrolase 23 to facilitate Puccinia striiformis f. sp. tritici infection (Yang et al. 2020). The majority of $\mathrm{R}$ proteins contain a nucleotide binding site (NBS) and leucine-rich repeat domains. The NBS domain of $\mathrm{R}$ proteins is a conserved region that is responsible for the binding and hydrolysis of ATP or GTP, which is essential for their biochemical activity (Ameline-Torregrosa et al. 2007; Tameling et al. 2002). The NBS region is involved in signal transduction cascades involving phosphorylation/dephosphorylation with GTP or ATP (Liu et al. 2007). ETI is qualitatively stronger and faster and is often characterized by a HR, but we generally need a better understanding of both PTI and ETI defense responses (Dodds and Rathjen 2010; Jones and Dangl 2006; Landoni et al. 2013). Both biotic and abiotic stresses can induce the release of ATP, which can also serve as a PAMP recognized by plants (Dodds and Rathjen 2010; Tanaka et al. 2014). ATP plays an important role in ETI defense response, but how the ATP-releasing systems are regulated by environmental stresses remains unclear (Tanaka et al. 2014).

ATP is synthesized or hydrolyzed by cpATPase, utilizing or generating a proton gradient by $\mathrm{H}^{+}$translocation. The cpATPase involved in photosynthesis is responsible for the synthesis of ATP from ADP in a process driven by the transthylakoid proton gradient (Kangasjärvi et al. 2014; Kramer et al. 2004). Of cpATPase's two domains, the $\mathrm{CFl}$ domain contains five subunits, denoted $\alpha$ (AtpA), $\beta$ (AtpB), $\gamma$ (AtpC), $\delta$ (AtpD), and $\varepsilon$ (AtpE) (von Ballmoos et al. 2009). ATP binding sites located in $\alpha$ - and $\beta$-subunits function in regulation and catalysis, respectively, and their conformational changes result in the synthesis/hydrolysis of ATP (Leyva et al. 2003). In particular, the AtpA subunit plays a pivotal role in plant energy metabolism.

Photosynthetic activity significantly affects the redox status and energy state by supplying ATP, NADPH, and carbon skeletons, which provide a basis for plant growth and trigger responses against external stress and their maintenance (Kangasjärvi et al. 2014). Plants have evolved multiple mechanisms to optimize photosynthesis under environmental fluctuations in order to balance growth with metabolism during costly defense responses (Kangasjärvi et al. 2014). Previous findings indicate that disease resistance is related to photosynthetic parameters, such as wheat Fusarium head blight, Jujube witches' broom disease, and powdery mildew in cucumber (Lindenthal et al. 2005; Xue et al. 2018; Yang et al. 2016). However, significant uncertainties remain in our understanding of how AtpA as a photosynthetic parameter is involved in stress response to pathogens and abiotic stress. Since AtpA was found enriched in nonfungus-infested leaves of mite-infested maize by leaf proteome analysis in maize, it suggests that a higher content of ATP could be required to improve stress tolerance in leaf of maize, and by extension perhaps plant resistance against enemies (Dworak et al. 2016). Furthermore, AtpA was accumulated under a flg22 treatment, a well-characterized PAMP, which is a peptide derived from the bacterial motor protein flagellin (Göhre et al. 2012). Song et al. (2014) reported that $a t p A$ was upregulated 5.01-fold in poplar
(Populus simonii) plants after $6 \mathrm{~h}$ of imposed heat stress, suggesting that this gene might play important roles in photosynthesis under conditions of heat stress. In other work, the relative expression of photosynthetic complex protein AtpA was increased under saline conditions in Desmostachya bipinnata; hence, accumulating more AtpA may promote the synthesis of ATP to meet the greater energy demand for sustained salt resistance in plants (Asrar et al. 2017). Despite its expression patterns being well known during different stress conditions, AtpA's biological functioning and mechanism(s) remain poorly understood. In this study, gene expression of atpA in tomato was induced significantly by B. cinerea and/or Clonostachys rosea at $96 \mathrm{~h}$ posttreatment (Supplementary Fig. S1).

Various signaling molecules, such as reactive oxygen species (ROS), nitric oxide (NO), hydrogen peroxide $\left(\mathrm{H}_{2} \mathrm{O}_{2}\right)$, and $\mathrm{Ca}^{2+}$, are involved in the regulation of multiple pathways controlling plant stress responses (Govrin and Levine 2000; Seifi et al. 2013). Antioxidant enzymes, such as catalase (CAT), superoxide dismutase (SOD), peroxidase (POD), and phenylalanine ammonia lyase (PAL), are crucial for plants' resistance against $B$. cinerea infection (Bui et al. 2019; Govrin and Levine 2000; Jing-Yu et al. 2020). To evaluate the physiological effects and functional mechanisms of atpA on B. cinerea resistance, we compared changes in ATP and ROS content, activity of ATP synthase and antioxidant enzymes, resistance-related genes, $\mathrm{Ca}^{2+}$ and $\mathrm{H}^{+}$levels, and chloroplast structure in transgenic and control tobacco plants. These results broaden our understanding of the resistance mechanisms against $B$. cinerea and broad-spectrum resistance as mediated by AtpA. Furthermore, these findings could potentially be useful for improving disease resistance traits in tomato cultivars.

\section{MATERIALS AND METHODS}

B. cinerea strains, plant material, and growth conditions. The $B$. cinerea strain used was isolated from a diseased tomato plant (Wang 2013; Wang et al. 2019) and cultured on potato dextrose agar plates at $25^{\circ} \mathrm{C}$ for 7 days. Conidial suspensions of the strain were prepared as previously described (Gong et al. 2017).

Seeds of homozygous tomato variety 09888-6 and cultivated tobacco K326 were provided by the Northeast Agricultural University Tomato Research Institute in China. The tomatoes were grown in a mixture of perlite/vermiculite/plant ash (1:6:2) in growth chambers at $25^{\circ} \mathrm{C} / 22^{\circ} \mathrm{C}$ (day/night) and $75 \%$ relative humidity under a 16-h/8-h (day/night) photoperiod. Tobacco seeds were germinated on Murashige and Skoog (MS) medium (M519; PhytoTechnology Laboratories, Shawnee Mission, KS). Seedlings were grown at $25^{\circ} \mathrm{C} / 22^{\circ} \mathrm{C}$ (day/night) and $60 \%$ relative humidity under a 16-h/8-h photoperiod in a phytotron. When their fifth leaves were fully developed, the tobacco plants were ready for use in the Agrobacterium-mediated transformations.

Cloning and sequencing of $\boldsymbol{a t p} \boldsymbol{A}$. Total RNA was extracted from tomato leaves and stems using TRIzol reagent (Invitrogen, Carlsbad, CA) according to the manufacturer's instructions, and then treated with RNase-free DNase I to remove any contaminating genomic DNA. First-strand cDNA was synthesized using $2 \mu \mathrm{g}$ of RNA with the RevertAid First-Strand cDNA Synthesis Kit (Thermo Fisher Scientific Co., Ltd., Shanghai, China). The full-length sequence of atpA was amplified from the cDNA by using forward primer 5' -ATGGTAACCATTCGAGCTGACG-3' and reverse primer 5'-TTATGCTTGTTCCTGAAGTATAAAACG-3'. The PCR amplification products were inserted into the pMD19-T vector (Takara, Dalian, China). The construct was then introduced into chemically competent Trans $5 \alpha$ Escherichia coli cells (TransGen Biotech, Beijing, China) according to the manufacturer's instructions and verified by sequencing.

Agrobacterium-mediated transformation of tobacco plants. The full-length sequence $(1,524 \mathrm{bp})$ of tomato atpA was inserted into the $\mathrm{pCAMh}+\mathrm{p}$ expression vector (structured by $\mathrm{pBI} 121$ and 
pCAMBIA2300) driven by the Cauliflower mosaic virus 35S (35SCaMV) promoter between BamHI and SacI (Supplementary Table $\mathrm{S} 1)$. pCAMh+p-atpA was introduced into A. tumefaciens strain LBA4404 by a freeze-thaw method. Transgenic tobacco plants were generated by a Agrobacterium-mediated leaf-disc transformation process (Horsch et al. 1985). DNA and RNA obtained from the $\mathrm{T}_{0} 100 \mathrm{mg} / \mathrm{liter}$ of kanamycin-resistant tobacco and untransformed tobacco (the wild type [WT]) were extracted for PCR and quantitative reverse transcription PCR (qRT-PCR) examinations (Supplementary Fig. S2). Genomic DNA was isolated from the tobacco leaves using a modified cetrimonium bromide method (Murray and Thompson 1980). The $\mathrm{T}_{0}$ plants were self-fertilized and the progeny obtained from the $T_{1}$ generation was named $T_{2}$. qRT-PCR results showed that all $100 \mathrm{mg} / \mathrm{liter}$ of kanamycinresistant plants had significantly higher mRNA expression levels than their WT counterparts. The $T_{2}$ generation of transgenic tobacco was considered homozygous (Wang et al. 2014). The $T_{2}$ seeds were then germinated on MS medium without any growth regulators or antibiotics; the plants were used for physiological and molecular analyses (Jing et al. 2015) to further estimate plant resistance to $B$. cinerea.

Evaluation of $B$. cinerea tolerance. Leaves of 1-month-old seedlings of transgenic lines and WT tobacco were inoculated with the $B$. cinerea strain using a modified version of the method described in Munis et al. (2010). A small wound (2 to $3 \mathrm{~mm}$ in length) was gently made in the third mature leaves with blades, to which $30 \mu \mathrm{l}$ of filtered spores $\left(10^{7} \mathrm{spore} / \mathrm{ml}\right)$ was applied per plant. These inoculated plants were kept in humid and dark conditions at $22^{\circ} \mathrm{C}$ for 30 days postinoculation (dpi).

In the whole-plant inoculation assay, transgenic and WT tobacco individuals were infected with $B$. cinerea through a suspension $\left(10^{7}\right.$ spores/ml)-spraying process until the plants were covered with droplets, whereas the control group was sprayed with only sterile water. For each treatment, a triplicate of 20 tobacco plants was used, all incubated under humid conditions at $22^{\circ} \mathrm{C}$. The disease index and the disease incidence of gray mold disease were each calculated at $15 \mathrm{dpi}$ according to Gong et al. (2017), albeit with slight modifications. The degree of disease severity after inoculation with $B$. cinerea was scored as follows: 0 , no lesion; 1 , a few scattered lesions covering $<2 \%$ of the tobacco leaf area; 2 , extensive lesions covering $>2 \%$ but $<5 \%$ of the tobacco leaf area; 3 , extensive lesions covering $>5 \%$ but $<25 \%$ of the tobacco leaf area, and; 4 , extensive lesions covering $>25 \%$ of the tobacco leaf area. The disease index for each treatment was calculated using the following formula:

$$
\text { Disease index }=\left\{\left(\sum_{i=0}^{4} \mathrm{~N}_{\mathrm{i}} \times \mathrm{i}\right) /\left(4 \times \sum_{i=0}^{4} \mathrm{~N}_{\mathrm{i}}\right)\right\} \times 100 \%
$$

where $\mathrm{i}$ is the severity score ( 0 to 4 ), for which 4 is the highest level, and $\mathrm{Ni}$ is the number of tobaccos with a severity of $\mathrm{i}$. The disease incidence was calculated at $15 \mathrm{dpi}$ using the following formula:

Disease incidence $=$ (no. of tobacco plants diseased/total no.) $\times 100 \%$

Chloroplast ultrastructure under $B$. cinerea infection. Leaf samples (of whole-plant inoculations) were collected close to infected sites, from green parts at the edge of necrotic lesions (cut into 2 to $3 \mathrm{~mm}$ ). All samples were taken at $15 \mathrm{dpi}$. Samples were pinned onto $2.5 \%$ (vol/vol) glutaraldehyde and placed in a vacuum for $3 \mathrm{~h}$. The prefixed samples were washed three times with $0.1 \mathrm{M}$ of phosphate-buffered saline (PBS; pH 6.8) for 15 min each time. Next, the samples were briefly postfixed in $1 \%$ osmium tetroxide, then rinsed again in $0.1 \mathrm{M}$ of PBS (pH 6.8) three times for 15 min each time. These specimens were dehydrated with 50, 70, 90, and $100 \%$ ethanol and soaked in a mixture of ethanol and acetone (1:1) for $10 \mathrm{~min}$. The above steps were carried out at $4^{\circ} \mathrm{C}$. Then the samples were dehydrated with $100 \%$ acetone at room temperature, fixed in 100\% resin overnight, and embedded in Spurr's medium. Thin sections (50 to $60 \mathrm{~nm}$ ) were cut using an LKB-V ultramicrotome. Sections were dyed with lead citrate and uranium acetate, and each sample was observed under a transmission electron microscope (H-7650; Hitachi, Tokyo, Japan).

Measurements of physiological parameters and secondary messengers. Transgenic and WT tobacco plants were sprayed with a $B$. cinerea suspension, as described above. Their third mature leaves were collected at $0,0.5,3,6,12,24,48,72$, and $96 \mathrm{~h}$ postinoculation (hpi) and cleaned with sterile water. Defenserelated enzyme (CAT, SOD, POD, PAL) activity and secondary messengers ( $\mathrm{NO}, \mathrm{O}_{2-}, \mathrm{H}_{2} \mathrm{O}_{2}$ ) were measured using a commercial chemical assay kit (Nanjing Jiancheng Bioengineering Institute, Nanjing, China). ATP content and cpATPase enzyme activity were measured with a commercial chemical assay kit (Jiangsu Keming Biotechnology Institute, Suzhou, China) per the manufacturer's manual.

Chlorophyll was extracted from leaves following Arnon (1949), with minor modifications. Leaf disks (each $0.04 \mathrm{~g}$ ) were homogenized in $0.6 \mathrm{ml}$ of $95 \%$ acetone on ice and the ensuing homogenate was then centrifuged at $3,000 \times g$ for $10 \mathrm{~min}$. The supernatant was retained, to which $95 \%$ ethanol was added for a total volume of $5 \mathrm{ml}$. Absorbance was recorded at 663, 645, and $470 \mathrm{~nm}$.

Flux measurement of net $\mathrm{Ca}^{2+}$ and $\mathrm{H}^{+}$. Transgenic and WT tobacco plants were infected with a $B$. cinerea suspension as described above. The third mature leaves from 1-month-old seedlings of tobacco individuals were used for net $\mathrm{Ca}^{2+}$ and $\mathrm{H}^{+}$ flux measurement, performed with noninvasive microtest technology (NMT) (NMT100 Series, YoungerUSA LLC, Amherst, MA; Xuyue Sci. \& Tech. Co., Ltd., Beijing, China) at 0, 0.5, and $3 \mathrm{hpi}$, with four biological replicates per time point. Net fluxes of $\mathrm{H}^{+}$and $\mathrm{Ca}^{2+}$ were measured by moving microelectrodes repeatedly between two positions (5 and $35 \mu \mathrm{m})$ adjacent to the exposed tobacco leaf mesophyll cells and calculated by applying Fick's law of diffusion (Liu et al. 2017).

RNA sequencing and analysis. Transgenic and WT tobacco plants infected with $B$. cinerea suspension spraying as described above, with three biological replicates at each time point, were used for RNA sequencing (RNA-seq) and its subsequent analysis. The third mature leaves from 1-month-old tobacco seedlings were sampled at 0.5 and 3 hpi for total RNA extraction, from which $1.5 \mu \mathrm{g}$ of RNA per sample was used to construct the RNA libraries. Deep sequencing was performed on an Illumina Hi-Seq 2500 platform at Biomarker Technologies Corporation (Beijing, China). After any adaptor sequences were removed and the low-quality sequences were filtered out, the remaining clean reads from each sample were mapped onto the tobacco genome Ntab-K326 (https://solgenomics.net) by using the TopHat 2 tool and its default parameters (Kim et al. 2013). The fragments per kilobase of transcript per million mapped reads method was selected to calculate the normalized expression level data of each library. Differentially expressed genes (DEGs) were identified by the package DESeq2 as those with a fold change $\geq 2$ and a false discovery rate (FDR) $\leq 0.05$. Gene Ontology (GO) enrichment was implemented by the GOseq R package (Li et al. 2018), whereas the Kyoto Encyclopedia of Genes and Genomes (KEGG) database was used to analyze pathway enrichment (Wei et al. 2019). All analyses were completed on the BMK Cloud (http://www.biocloud.net; Biomarker Technologies Corporation).

Analysis of broad-spectrum resistance. Seed germination rates of WT and transgenic tobacco plants under different concentrations of salt stress $(0,150$, and $250 \mathrm{mM}$ of $\mathrm{NaCl})$ were counted at 0 to 15 days posttreatment. Likewise, growth assays of seedlings were compared between WT and transgenic lines under the same salinity treatments $(0,150$, and $250 \mathrm{mM}$ of $\mathrm{NaCl})$. For 
this, seeds were sown on MS medium for 7 days, after which the ensuing seedlings were moved to MS medium containing different concentrations of $\mathrm{NaCl}$ for 7 days. The 4-week-old seedlings were then moved into one-half-strength Hoagland culture medium differing in $\mathrm{NaCl}$ concentration (see above) for the growth assays.

Finally, for the leaf mold resistance analysis, 1-month-old seedlings of transgenic and WT tobacco were infected with Cladosporium fulvum (provided by the Northeast Agricultural University Tomato Research Institute) through suspension $\left(10^{7}\right.$ spores $/ \mathrm{ml}$ ) spraying and the phenotype was recorded at $15 \mathrm{dpi}$ (Xue et al. 2017). This was performed as described for the whole-plant B. cinerea inoculation assay above.

Statistical analyses. All data are presented as the mean \pm standard deviation. Statistical analysis was carried out with oneway analysis of variance (ANOVA) in SPSS software (SPSS, Chicago, IL). Separations were performed using Duncan's multiple range tests. Differences at $P<0.05$ were considered statistically significant and are represented by different letters. All experiments were repeated three times.

\section{RESULTS}

atpA cloning and sequence analysis. A 1,524-bp full-length cDNA of atpA, which encoded a peptide of 507 amino acids, was successfully amplified. There was one single nucleotide mutation (T674 $\rightarrow$ C674) compared with the chloroplast genomic sequence; this mutation was a single amino acid change (Val 225 $\rightarrow$ Ala 225). The amplified sequence was deposited in GenBank under accession number BankIt2317821 atpA MT134327.

Overexpression of atpA enhances plant resistance to B. cinerea. To further analyze the function of $a t p A$ in response to
$B$. cinerea, the full-length atpA sequence was cloned into the plant expression vector $\mathrm{pCAMh}+\mathrm{p}$ driven by the $35 \mathrm{~S}-\mathrm{CaMV}$ promoter and transformed into tobacco. These 31 lines were screened out by kanamycin-selection medium, PCR, and qRT-PCR assays to obtain the $\mathrm{T}_{0}$ generation of transgenic plants in total. Three atpA overexpression lines (OE17, OE21, and OE30) with higher expression levels of atpA were selected for further analysis (Supplementary Fig. S2).

The $B$. cinerea-treated WT tobacco plants showed severe symptoms, in the form of pronounced lesion development, which included obvious watery circular lesions with a yellowish and obscure boundary, at $7 \mathrm{dpi}$. No obvious symptoms or smaller range of browning around the leaf wound sites were observed in the transgenic plants (Fig. 1A). The WT leaves showed yellowing and appeared withered at 30 dpi. Necrotic lesions were smaller in the leaves of OE17, OE21, and OE30 than in those of WT (Fig. 1B), suggesting that the spread of necrotic lesions in OE17, OE21, and OE30 was controlled by atpA overexpression.

In the whole-plant inoculation assay, gray mold disease symptoms were significantly lower in all atpA-overexpressing tobacco plants compared with the WT. The disease incidence was $80,18.33,20$, and $23.33 \%$ in WT, OE17, OE21, and OE30 tobacco, respectively (Fig. 1C), with corresponding disease index values of $54.17,5,6.25$, and $7.5 \%$ (Fig. 1D). Overexpression of atpA significantly reduced both the disease index and the disease incidence in tobacco plants infected with $B$. cinerea.

Overexpression of atpA stabilized chloroplast structure under $B$. cinerea infection. To clarify whether the ultrastructure of chloroplasts is affected by overexpression of atpA under $B$. cinerea inoculation, infected leaves from the WT and transgenic tobacco seedlings were examined under an electron microscope for their green tissues around the necrotic lesions at infected sites. In the
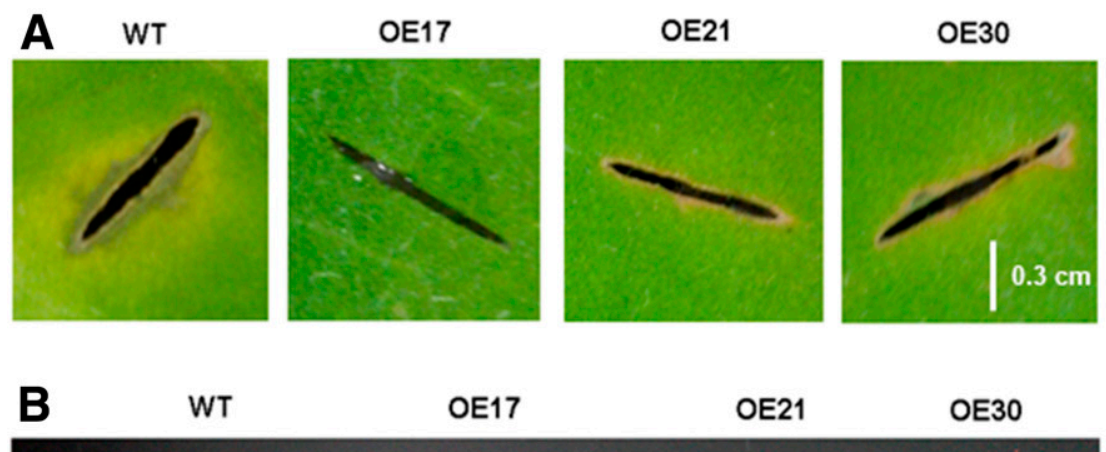

$$
\text { WT }
$$
OE17

$$
\text { OE21 }
$$

OE30

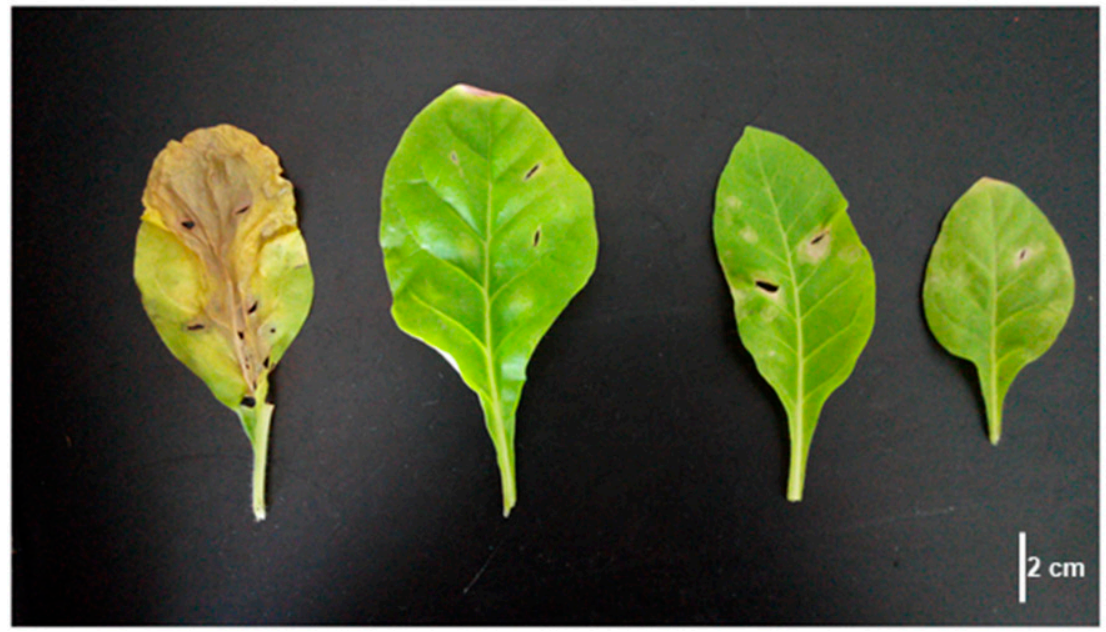

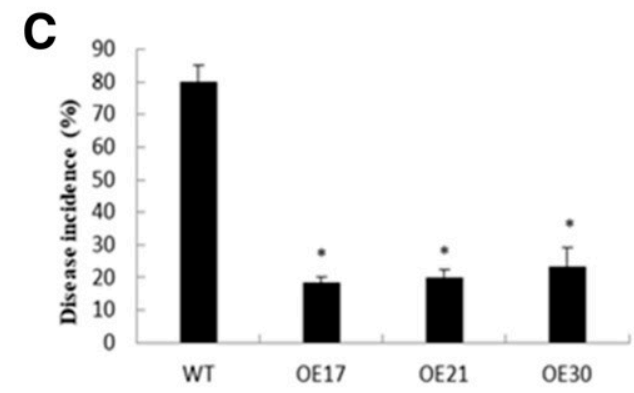

D

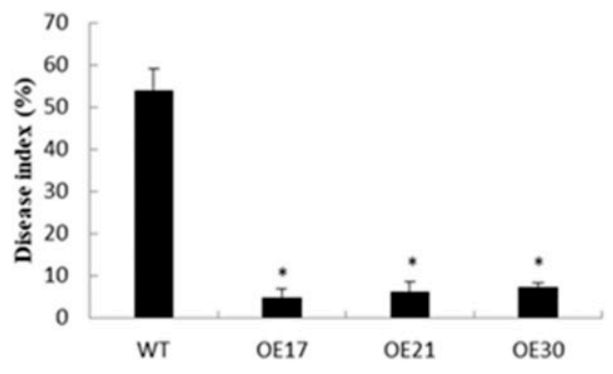

Fig. 1. Necrotic lesion area in leaves at $\mathbf{A}, 7$ days postinoculation (dpi) and $\mathbf{B}, 30$ dpi and $\mathbf{C}$, disease incidence and $\mathbf{D}$, disease index for gray mold disease after inoculations with Botrytis cinerea in wild-type (WT) and transgenic lines (OE17, OE21, and OE30) of tobacco plants. Error bars represent the standard deviation for three independent replicates. Significant differences between the transgenic lines and WT were analyzed by analysis of variance. Different letters indicate significant differences compared with the WT at $P<0.05$. 
absence of $B$. cinerea infection, the chloroplasts appeared elongated and regularly located along the cell wall. However, the chloroplasts were evidently swollen and more seriously damaged in the WT than transgenic tobacco plants at $15 \mathrm{dpi}$. In the WT samples, chloroplasts were approximately circular in shape, with the grana lamellae and stroma lamellae of thylakoid distributed loosely, with less lamella and disintegration in the chloroplasts. In addition, large osmiophilic plastoglobules had accumulated and membrane systems were degraded. These results indicated that atp $A$ overexpression stabilized the chloroplast structure under B. cinerea infection (Fig. 2).

Effect of infection on plant physiological parameters. To assess the potential effect of atpA overexpression on $B$. cinerea resistance, tobacco plant chlorophyll content, ROS levels, signal molecules, and enzyme activity were analyzed in leaves of the WT and OE17, OE21, and OE30. Chlorophyll $a$ contents were higher in OE17, OE21, and OE30 than in the WT during 12 to 48 hpi. Chlorophyll $b$ content was ascendant and higher in the WT than in OE17, OE21, or OE30 within 6 hpi. Yet chlorophyll (b) content increased rapidly in transgenic plants at $12 \mathrm{hpi}$ and peaked at $48 \mathrm{hpi}$, at which time it surpassed that in the WT (Supplementary Fig. S3; Supplementary Table S2).

CAT activity increased before 3 hpi in all tobacco plants, but especially in the transgenic lines. At $12 \mathrm{hpi}$, CAT activity in OE17, OE21, and OE30 exceeded that in the WT (Fig. 3A). SOD activity decreased in all tobacco plants at $0.5 \mathrm{hpi}$, but then exhibited a sharp increase at 3 hpi. A slight decrease was observed in transgenic lines at $12 \mathrm{hpi}$, followed by an increase to a level higher than that in the WT at 24, 48, and 96 hpi (Fig. 3B). Likewise, in all tobacco, POD activity first decreased but then increased by $96 \mathrm{hpi}$. In the transgenic lines, POD activity began to increase at $72 \mathrm{hpi}$ and reached maximum values at $96 \mathrm{hpi}$ to levels 2.54-, 3.51-, and 2.54fold higher than in the WT (Fig. 3C). PAL activity increased at 3 hpi in all three transgenic lines to levels slightly higher than that in the WT; the latter's PAL activity declined to a greater extent at 12 hpi, whereas PAL activity was higher in the transgenic lines, and significantly so at 6 and 96 hpi (Fig. 3D; Supplementary Table S3).
F1F0-ATPase activity was higher in transgenic lines during the whole-plant infection period than in the WT. Compared with the WT, early activity of F1F0-ATPase in the transgenic lines increased considerably over time yet it decreased markedly at $12 \mathrm{hpi}$, although it was still higher (Fig. 3E). F1F0-ATPase activity of the transgenic lines reached its maximum value at 3 hpi to levels 1.93-, 1.82-, and 1.57-fold higher than the WT. Significantly increased ATP content was observed at $0.5 \mathrm{hpi}$, which peaked at $3 \mathrm{hpi}$ to levels 3.70- to 4.41 -fold greater than in the WT. ATP content began to decline at $6 \mathrm{hpi}$ in the WT, becoming 8.00 - to 12.00 -fold lower than that in the transgenic lines. ATP content decreased in transgenic lines at $12 \mathrm{hpi}$ and began to increase at $24 \mathrm{hpi}$, so that it exceeded that of the WT as time elapsed (Fig. 3F).

$\mathrm{H}_{2} \mathrm{O}_{2}$ content in the transgenic lines began to increase at $0.5 \mathrm{hpi}$ and peaked at $3 \mathrm{hpi}$, whereas it declined in the WT during the postinoculation period. The greatest difference in $\mathrm{H}_{2} \mathrm{O}_{2}$ content between the transgenic lines and the WT was observed at 3 hpi when the transgenic lines/WT ratio of 3.35 to $4.37: 1$ was maximal. $\mathrm{H}_{2} \mathrm{O}_{2}$ content increased in the WT thereafter at 6 and 12 hpi but remained lower than that in the transgenic tobacco (Supplementary Fig. S4A; Supplementary Table S4). The NO content of WT decreased sharply at 0.5 hpi to a level significantly lower than that of the transgenic lines. At 12 hpi, however, the NO level increased markedly in transgenic lines and was maintained at relatively high levels until it sharply declined at 24 and $48 \mathrm{hpi}$. The NO level increased rapidly again in transgenic lines at $72 \mathrm{hpi}$, whereas the NO level in the WT was still strongly inhibited (Supplementary Fig. S4B). $\mathrm{O}_{2-}$ content increased steeply in transgenic plants at $48 \mathrm{hpi}$, attaining levels 2.5 to 3.21-fold higher than in the WT. Furthermore, after 48 hpi, $\mathrm{O}_{\overline{2}}$ content in OE17, OE21, and OE30 lines continued to exceed that in the WT (Supplementary Fig. S4C).

Effects on $\mathrm{Ca}^{2+}$ and $\mathrm{H}^{+}$flux in cells of the mesophyll. NMT was used to investigate the dynamic change of $\mathrm{Ca}^{2+}$ and $\mathrm{H}^{+}$ flux in mesophyll cells in living tobacco leaves (third from the top of the plant). $\mathrm{Ca}^{2+}$ was characterized by slight efflux under normal conditions, with no sharp distinction between WT and transgenic tobacco plants. A faster decline in $\mathrm{Ca}^{2+}$ efflux in transgenic tobacco was observed compared with the WT at $0.5 \mathrm{hpi}$,

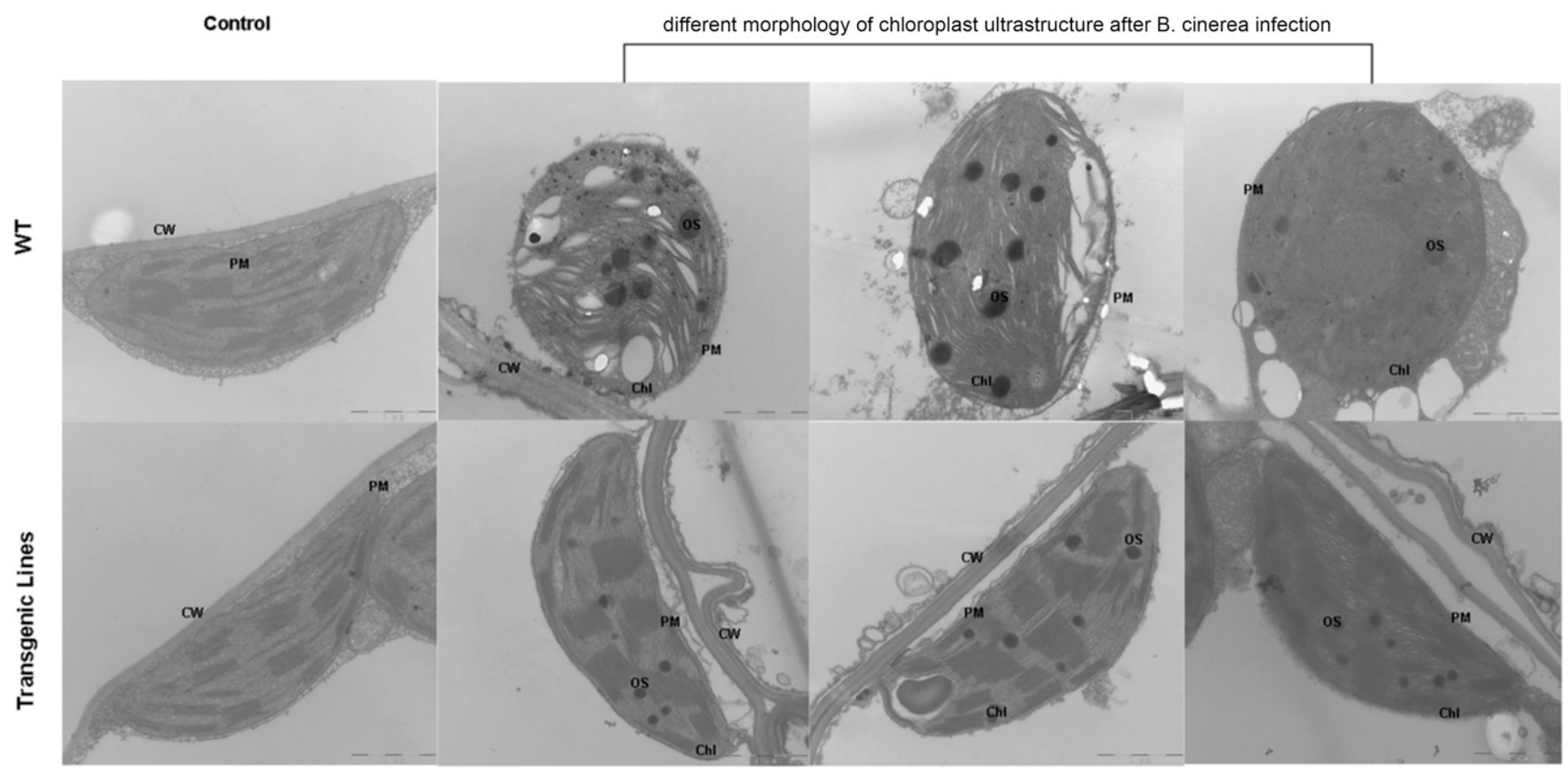

Transmission electron microscopy, $\times 30000$

CW: Cell wall

PM: plasma membrane

Chl: chloroplast

OS: Osmiophilic granule

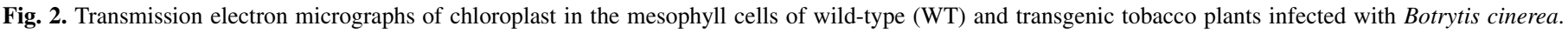
Magnification $\times 30,000 . \mathrm{CW}=$ cell wall, $\mathrm{PM}=$ plasma membrane, $\mathrm{Chl}=$ cholorplast, and $\mathrm{OS}=$ osmiophilic granule. 
whereas total $\mathrm{Ca}^{2+}$ efflux was significantly greater in the transgenic lines of tobacco than in the WT at 3 hpi (Fig. 4A, B, and C). For $\mathrm{H}^{+}$ efflux, it was slightly higher in the WT than in transgenic tobacco under normal growing conditions. After $B$. cinerea infection, the content of $\mathrm{H}^{+}$efflux in transgenic plants slightly increased at $0.5 \mathrm{hpi}$, yet no significant differences were detected among the tobacco plants. Evidently, $\mathrm{H}^{+}$efflux in transgenic lines remained stable throughout the $B$. cinerea treatment period, whereas a decreasing trend was noticeable in the WT at 3 hpi (Fig. 4D, E, and F).

Transcriptome sequencing and correlation analysis. Given the physiological characteristics of WT and transgenic tobacco plants under $B$. cinerea infection, transcription level changes in tobacco were estimated. The raw sequence data reported in this study were deposited in the Genome Sequence Archive at the Beijing Institute of Genomics of the Chinese Academy of Sciences, under accession number CRA002377 (publicly available at https:// bigd.big.ac.cn/gsa).

The bar charts in Figure 5 convey the number of up- and downregulated DEGs found between atpA overexpression lines and the WT at different time points after $B$. cinerea infection. Far more downregulated and upregulated DEGs were detected at $0.5 \mathrm{hpi}$ (3,961 and 3,463, respectively) than at 0 hpi $(2,084$ and 1,441, respectively). The number of upregulated DEGs at $3 \mathrm{hpi}$ decreased to 1,612, whereas the downregulated DEGs decreased to 2,408 (Fig. 5A). Two-way ANOVAs were used for robust statistical analysis of the RNA-seq data for the factors of genotype (WT versus atpA overexpression) and treatment (control versus $B$. cinerea inoculation). Overall, 5,421 genes were significantly affected by $B$. cinerea inoculation (having significant differential expression between $B$. cinerea-inoculated and inoculated WT plants; FDR $\leq 0.05$ ); 3,530 were significantly affected by atpA overexpression, with 9,855 genes found significantly influenced by the interaction between treatment and genotype (i.e., had significant differential expression between the $B$. cinerea-inoculated WT and $B$. cinereainoculated atpA-overexpressing plants; FDR $\leq 0.05$; Fig. 5B). Hierarchical clustering was then carried out to discern the differences and similarities among the $B$. cinerea-responsive DEGs at various time points in the WT and atpA overexpression tobacco, in a visualized way (Supplementary Fig. S5). The gene expression profiles between the WT and transgenic lines differed significantly among the time points during $B$. cinerea infection.

All unigenes were assessed in the GO database. The results indicated that most transcripts were enriched in the cellular process (GO: 0009987), metabolic process (GO: 0008152), singleorganism process (GO: 0044699), response to a stimulus (GO: 0050896) within the biological process category, cell part (GO: 0044464), cell (GO: 0005623), organelle (GO: 0043226) within the
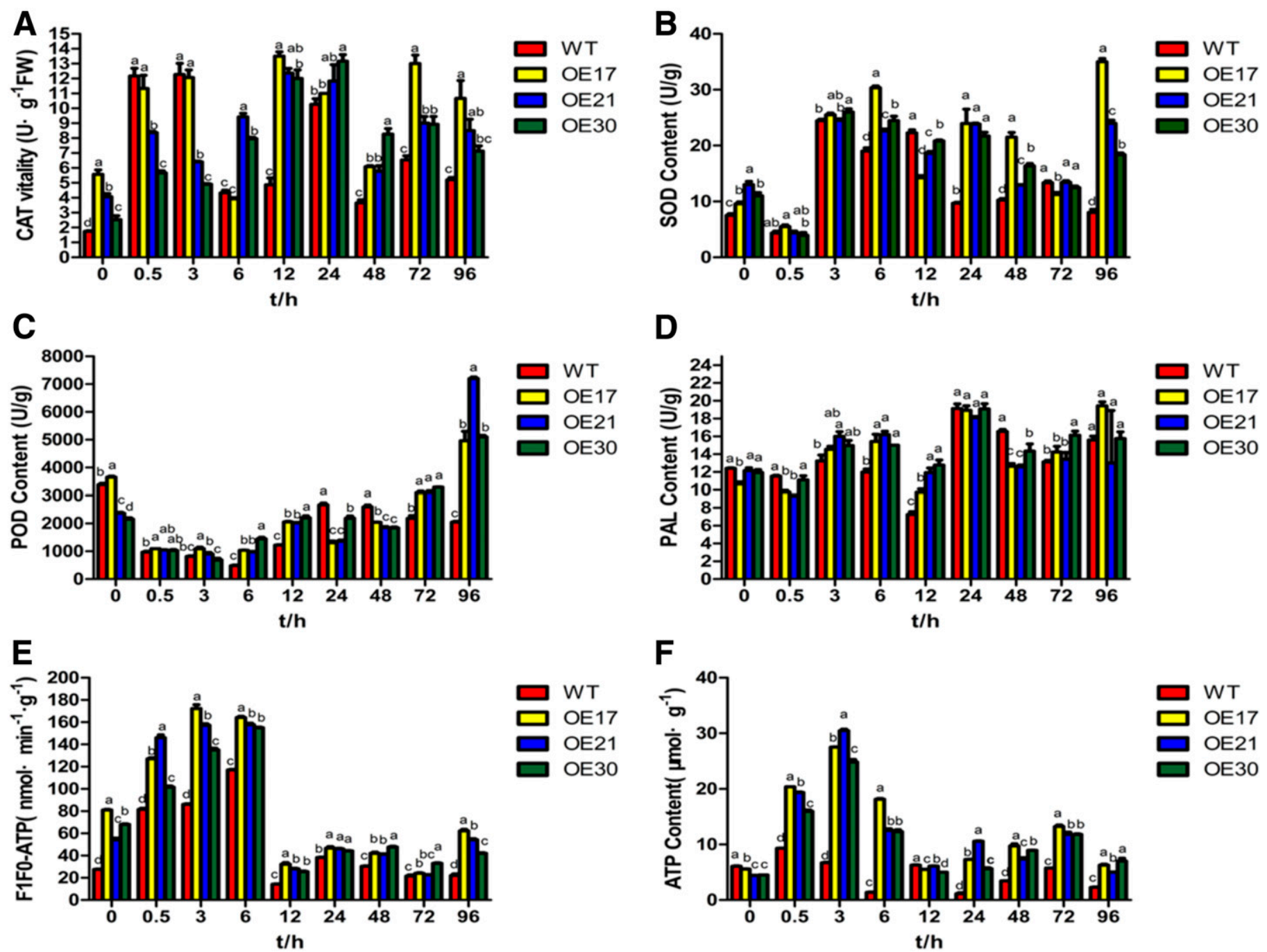

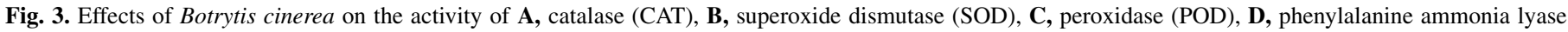

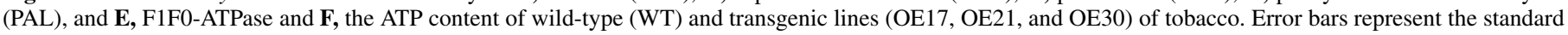

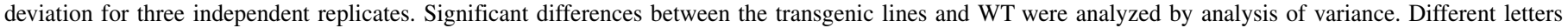
indicate significant differences compared with the WT at $P<0.05$. 
cellular component category, and binding (GO: 0005488) and catalytic activity (GO: 0003824) within the molecular function category (Fig. 6A and B; Supplementary Table S5). More functional terms were found for biological process, however.

We carried out the KEGG annotations separately at $0,0.5$, and 3 hpi (Fig. 6C, D, and E; Supplementary Tables S6, S7, and S8). At
$0.5 \mathrm{hpi}$, the top $20 \mathrm{KEGG}$ enrichments clearly indicated that the DEGs were enriched in the following: circadian rhythm-plant (ko04712), pentose phosphate pathway (ko00030), terpenoid backbone biosynthesis (ko00900), carbon metabolism (ko01200), glutathione metabolism (ko00480), glyoxylate and dicarboxylate metabolism (ko00630), carotenoid biosynthesis (ko00906),

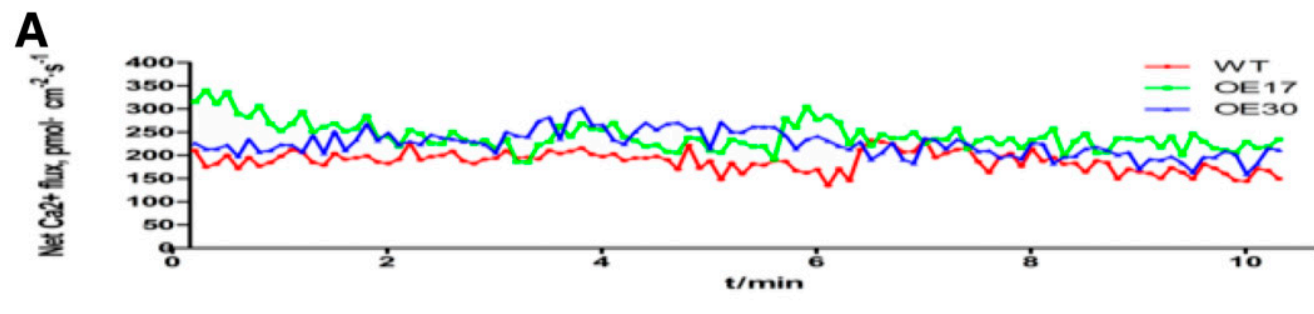

B
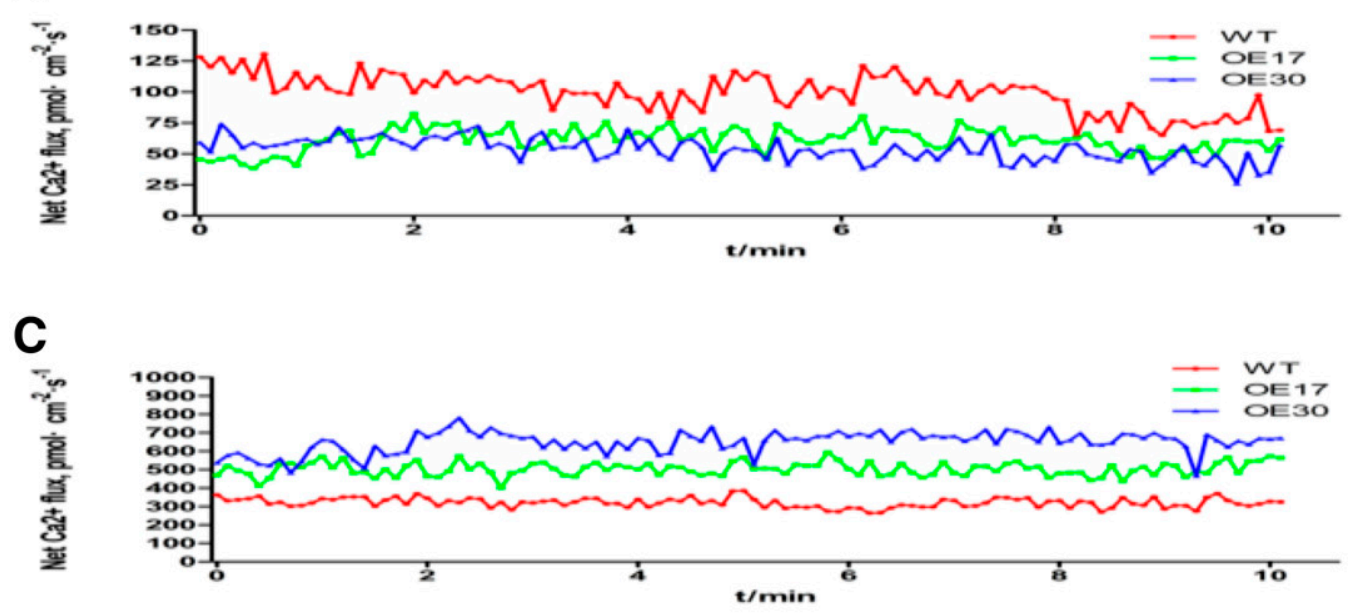

D

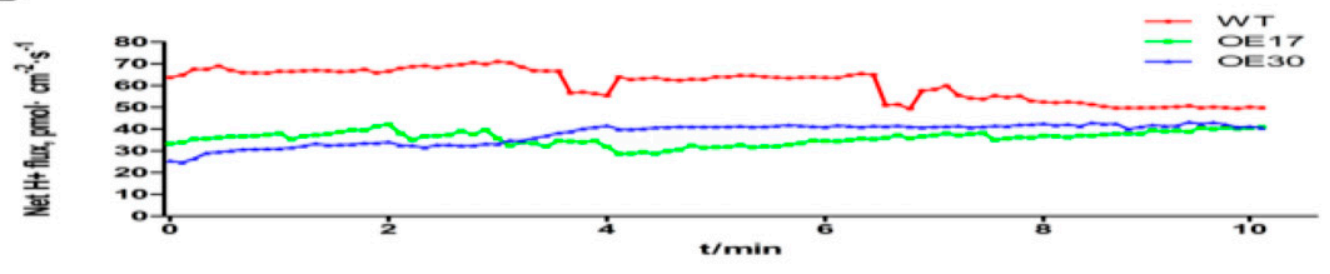

E

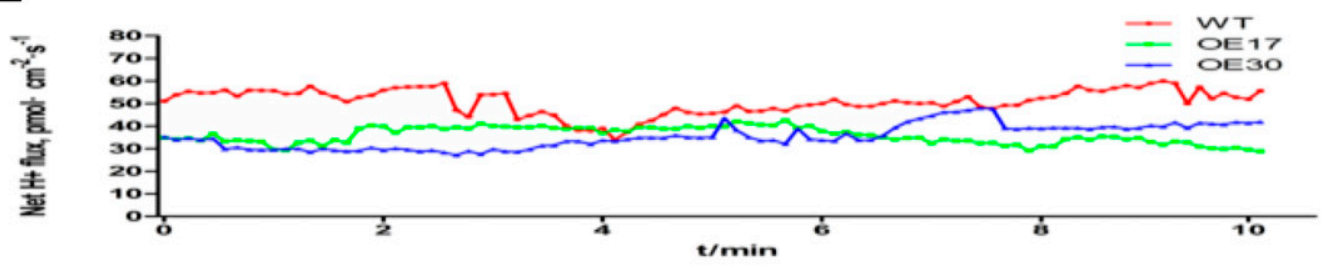

$\mathbf{F}$

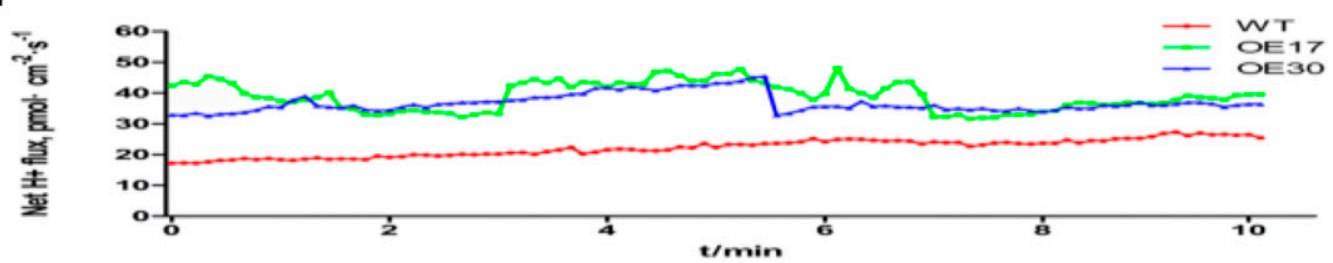

Fig. 4. Transient net $\mathbf{A}, \mathbf{B}$, and $\mathbf{C}, \mathrm{Ca}^{2+}$ and $\mathbf{D}, \mathbf{E}$, and $\mathbf{F}, \mathrm{H}^{+}$flux responses to Botrytis cinerea for various times in mesophyll cells of wild-type (WT) and transgenic lines (OE17 and OE30) of tobacco. In A and D, the tobacco plants were treated with buffer (mock-inoculated treatment). In B and E, they were treated with $B$. cinerea for $0.5 \mathrm{~h}$ postinoculation (hpi). In $\mathrm{C}$ and $\mathrm{F}$, they were treated with $B$. cinerea for $3 \mathrm{hpi}$. Steady-state flux profiles of $\mathrm{Ca}^{2+}$ and $\mathrm{H}^{+}$were examined by continuous flux recordings (about $10 \mathrm{~min}$ ). Positive values represent the $\mathrm{Ca}^{2+}, \mathrm{H}^{+}$efflux. 
glycolysis/gluconeogenesis (ko00010), photosynthesis (ko00195), and porphyrin and chlorophyll metabolism (ko00860) (Fig. 6D; Supplementary Table S4). Later, at 3 hpi, the DEGs were highlighted in protein processing in the endoplasmic reticulum (ko04141), plant hormone signal transduction (ko04075), plant-pathogen interaction (ko04626), circadian rhythm (ko04712), and inositol phosphate metabolism (ko00562) (Fig. 6E; Supplementary Table S5). These results suggested that plants have evolved a range of defense mechanisms that predominate at different stages of fungal infection, and that infection by $B$. cinerea can lead to changes in many metabolic pathways mediated by atpA.

Analysis of broad-spectrum resistance/tolerance. To estimate whether overexpression of atpA in plants confers salt tolerance in them, both WTand transgenic tobacco were treated with differing concentrations of $\mathrm{NaCl}$. The transgenic lines exhibited a significantly better seed germination rate and seedling growth under salt stress than did the WT (Supplementary Fig. S6). In addition, the WT and transgenic tobacco plants were sprayed with a spore suspension to further investigate the function of atpA in response to Cladosporium fulvum; in this experiment, the transgenic tobacco showed increased resistance to Cladosporium fulvum (Supplementary Fig. S7).

\section{DISCUSSION}

AtpA, encoded by atpA, is a key protein for energy metabolism and multiple cell processes and was identified as specifically enriched in tomato when induced by Clonostachys rosea under $B$. cinerea infection. Hence, atpA may play a key role in tomato defense that is primed by Clonostachys rosea. In this study, atpA was cloned and validated for its function of resistance toward $B$. cinerea infection by heterologous expression of atp $A$ in Nicotiana tabacum. The isolated atpA gene has one single base that differs from the published sequence.

We found that expression of atpA greatly suppressed the gray mold disease index and disease incidence compared with the WT. That the visible lesions were significantly reduced in transgenic tobacco is a good morphologic indicator of the capability of atpA expression to foster plant resistance to $B$. cinerea. Moreover, our results indicated that characteristic HR-like necrotic lesions at the site of infiltration were evident and the lesions did not develop beyond the initial invaded area in atpA-overexpressing plants at 30 dpi. These results confirmed that atpA overexpression reinforces plant resistance to gray mold disease by inhibiting the ability of $B$. cinerea to utilize dead tissue to facilitate its further colonization of hosts. In short, atpA overexpression can prevent the fungus from disrupting the host's defense mechanism, an action key to its pathogenicity. Accordingly, corresponding effects of atpA overexpression on photosynthetic parameters, antioxidant systems, and gene expression under $B$. cinerea inoculation were demonstrated at the structural, physiological, and molecular levels.

In a previous study, infection by the stripe rust pathogen altered the thylakoid structure, therefore resulting in a reduction of photosystem II (PSII) activity in susceptible wheat plants (Chen et al. 2015). Other work found that B. cinerea was blocked in the area of completely destroyed Mesembryanthemum crystallinum cells, and no hyphae were found in the vicinity of leaf lesions (Gabara et al. 2012). In our study, B. cinerea infection destroyed the structure of chloroplasts, thereby likely lowering PSII activity in the WT. Excessive damage to the structure of plant chloroplasts significantly weakened their photosynthesis and this reduced the available supply of ATP. However, the chloroplast ultrastructure in the cells surrounding the necrotic lesions could be maintained with overexpression of atpA to confer resistance by tightly inhibiting the HR-like lesions and the pathogen-induced senescence of leaf tissue.

Furthermore, atpA-overexpressing transgenic tobacco plants had higher cpATPase activity and greater ATP content during the early stage of $B$. cinerea infection. Kanazawa et al. (2017) confirmed a decisive role for ATP synthase regulation in maintaining photosynthetic control of electron transfer to prevent photodamage via modulation of the thylakoid proton motive force, whereas Takagi et al. (2017) showed that cpATPase regulates $\mathrm{H}^{+}$efflux activity in response to such changed drivers of photosynthesis. Consistent with those findings, we found that $\mathrm{H}^{+}$efflux was stabilized at a relatively high level at $3 \mathrm{hpi}$, a result consistent with the high cpATPase levels we observed in transgenic tobacco. It is conceivable that atpA plays a crucial role in stabilizing chloroplast ultrastructure and $\mathrm{H}^{+}$efflux and also enhances cpATPase activity, which together sustain sufficient photosynthesis to produce adequate ATP to cope with the $B$. cinerea infection. Buchert et al. (2012) confirmed that a change in ATP synthase activity is able to minimize ROS-promoted photoinhibition. ATP is vital for ensuring resistance to $B$. cinerea.

A

B
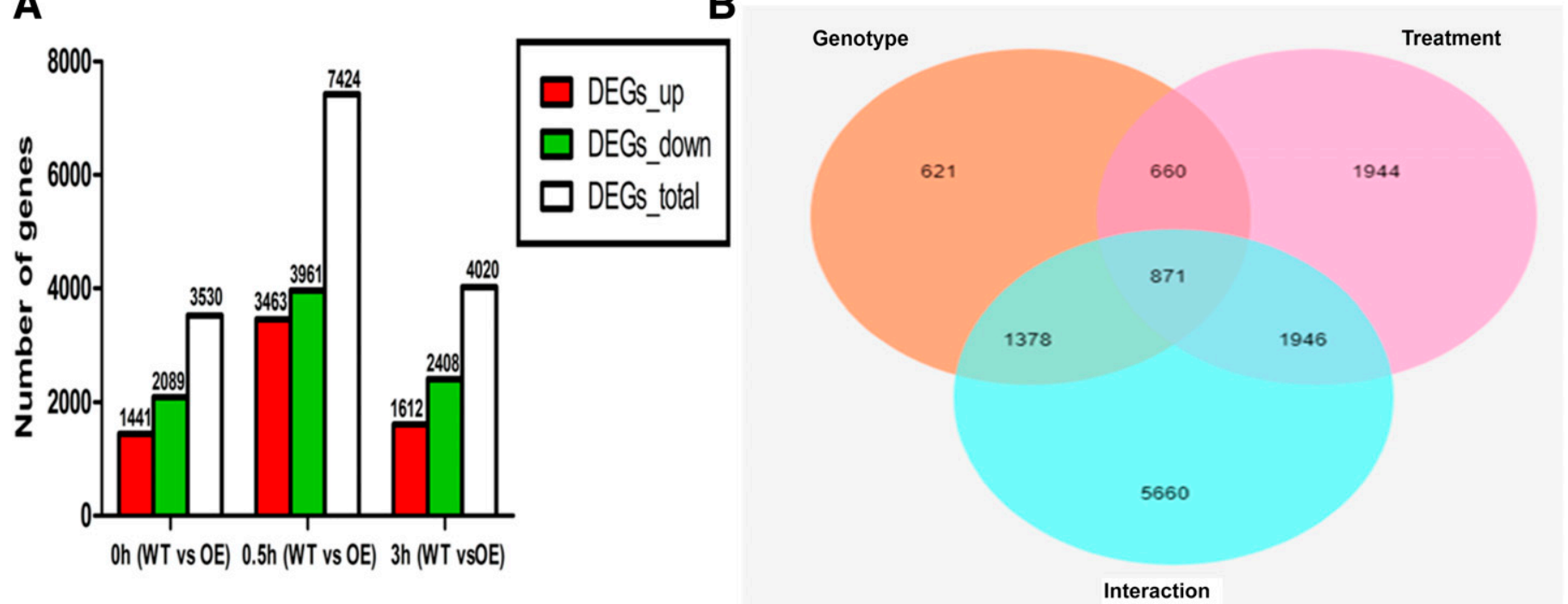

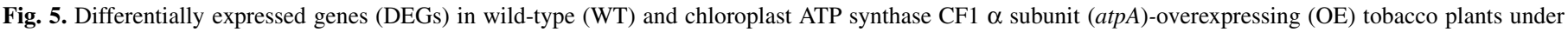

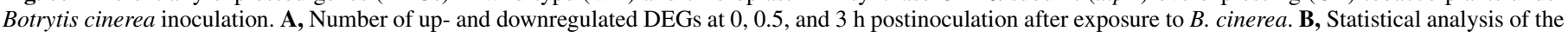

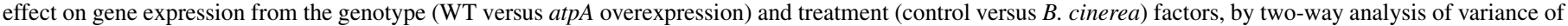
the RNA sequencing data. The numbers of genes showing a significant change (false discovered rate-adjusted $P<0.05$ ) are displayed in the Venn diagram. 


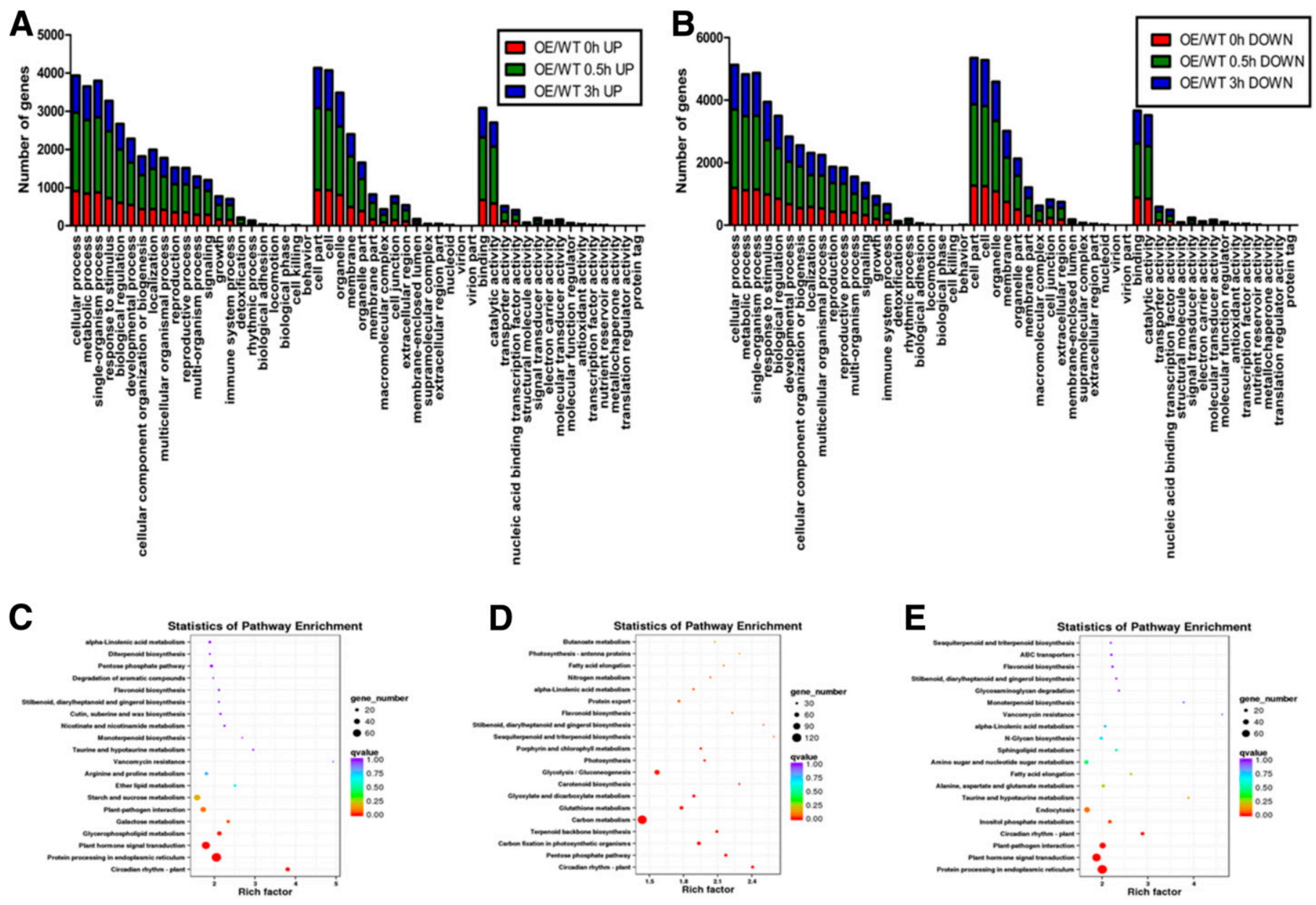

Fig. 6. A and B, Comparative Gene Ontology enrichment analysis and C, D, and E, Kyoto Encyclopedia of Genes and Genomes pathway analysis at 0 , 0.5 , and 3 days postinoculation (dpi) of the differentially expressed genes (DEGs). The $x$-axis indicates the enrichment factor on a scale from 0 to 2.4 . Dot color and size indicate the $\mathrm{Q}$ value and gene number, respectively, as shown on the keys to the right of the lower panels. OE $=$ overexpressing and WT $=$ wild type.

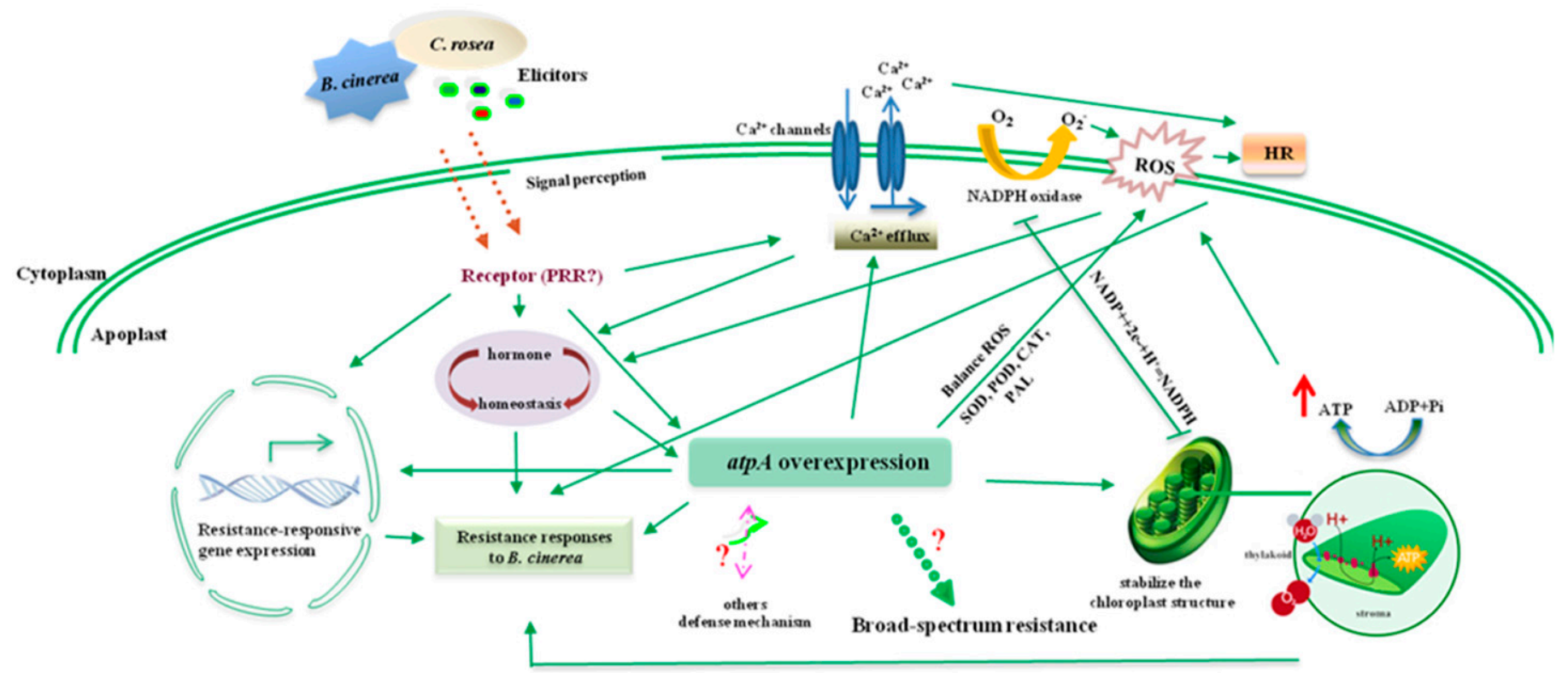

Fig. 7. The proposed mechanism of plant defense response as mediated by the chloroplast ATP synthase CF1 $\alpha$ subunit (AtpA). Arrows indicate positive regulation. $\mathrm{PRR}=$ pattern recognition receptor, $\mathrm{ROS}=$ reactive oxygen species, $\mathrm{HR}=$ hypersensitive response, $\mathrm{SOD}=$ superoxide dismutase, $\mathrm{POD}=$ peroxidase, $\mathrm{CAT}=$ catalase, and $\mathrm{PAL}=$ phenylalanine ammonia lyase . 
Moreover, ATP has been reported to function as a signal that can modulate various metabolic functions (Hao et al. 2012); in addition, ATP may be recognized by P2K1, a plasma membrane-localized purinergic receptor, which can elevate cytosolic free $\mathrm{Ca}^{2+}$ concentrations (Tanaka et al. 2014), leading to the production of NO or ROS (Demidchik et al. 2009; Song et al. 2006). In our study, both $\mathrm{NO}$ content and $\mathrm{H}_{2} \mathrm{O}_{2}$ content were increased by atpA overexpression under $B$. cinerea infection. Higher $\mathrm{H}_{2} \mathrm{O}_{2}$ content can protect cells of leaf veins from fungal invasion (Gabara et al. 2012), whereas NO is critically involved in fungal physiology and development and fungal plant infection (Zhao et al. 2020). Furthermore, NO accumulation figures prominently in $\beta$-aminobutyric acid-induced resistance against $B$. cinerea in tomato plants (Li et al. 2020).

$\mathrm{H}_{2} \mathrm{O}_{2}, \mathrm{NO}$, and $\mathrm{Ca}^{2+}$ are significant signaling molecules in plants that trigger multiple cellular responses that induce the expression of defense-related genes under $B$. cinerea infection. We found that ROS (i.e., $\mathrm{O}_{2}^{-}$and $\mathrm{H}_{2} \mathrm{O}_{2}$ ) and $\mathrm{Ca}^{2+}$ generation increased rapidly in transgenic plants treated with $B$. cinerea, a response that could have fostered the defense-associated HR within a short time. Consistent with our results, an earlier study showed that $\mathrm{Ca}^{2+}$ efflux was an essential factor causing the HR of $N$. benthamiana to Pseudomonas syringae (Nemchinov et al. 2008).

Defense enzymes are related to the biosynthesis of signaling molecules and are important to plant disease resistance. We observed that overexpressing atpA could tightly control the HR-like lesion, inhibiting the formation of gray mold, and it significantly enhanced CAT, SOD, POD, and PAL enzyme activity in host tobacco leaves. This result suggests that atpA overexpression enables plants to avoid oxidative damages more effectively than can WT counterparts.

The coregulation of carbohydrate metabolism and photosynthesis with defense mechanisms is a general plant response to an invading pathogen (Gabara et al. 2012). Fungal pathogen infection is closely correlated with changes in metabolic pathways in host plant tissues such as photosynthesis. The leaf organ, as a "source" tissue, functions to synthesize energy-rich molecules via photosynthesis for the transport of carbon (Xue et al. 2018). It is noteworthy that atpA overexpression promoted the expression of genes related to carbon metabolism, photosynthesis, the pentose phosphate pathway, and both porphyrin and chlorophyll metabolism, all of which belong to sucrose synthesis at $0.5 \mathrm{hpi}$; at $3 \mathrm{hpi}$, the DEGs were involved in protein processing, hormone signal transduction, plant-pathogen interaction, and metabolism. These results suggest that atpA can regulate the genes involved in those pathways central for synthesizing more energy and sources early in the fungal infection process (at $0.5 \mathrm{hpi}$ ), after which it regulates the genes related to disease resistance reactions and signal transduction (at $3 \mathrm{hpi}$ ). We hypothesize that the resistance response mediated by AtpA is rapid and strong during the initial stage of infection, a critical stage for $B$. cinerea development. Other research has claimed that the cells surrounding an invaded area of leaf are maintained by a continuing supply of the tricarboxylic acid (TCA) cycle, conferring resistance by both tightly controlling the defenseassociated HR and slowing pathogen-induced senescence (Seifi et al. 2013). In addition, C: $\mathrm{N}$ metabolism in the plant host plays an important role in its resistance response against necrotrophic pathogens (Seifi et al. 2013). Consistent with this observation, we found that those genes involved in the TCA cycle and carbon metabolism were substantially upregulated in transgenic tobacco plants at 0.5 hpi.

Thus far, most studies have focused on pathogen-resistant genes or proteins. Moreover, few researchers have examined the effects of pathogenic infection on proteins related to vital photosynthetic parameters (Chen et al. 2015; Yang et al. 2016). This study is the first to find that AtpA may have critical biological functions in B. cinerea, in Cladosporium fulvum, and even in how plants may respond to $\mathrm{NaCl}$ stress. The results are generally consistent with a previous study claiming that overabundant AtpA may increase the synthesis of ATP to satisfy greater requirements of energy for persistent salt resistance (Asrar et al. 2017). Our study further supports two assertions about the process conferring plant resistance: it is complex, and one likely regulated by the interaction of multiple pathways (Fig. 7) (Gong et al. 2017; Mouekouba et al. 2014; Wang et al. 2019). Taken together, our results lead us to presume that both PTI and ETI are involved in AtpA-mediated resistance to $B$. cinerea, a view requiring more analysis in our future work. This study nonetheless provides a starting point to clarify the molecular mechanism of broad-spectrum resistance of AtpA. Whether the atpA gene is generalizable to other species and field settings merits careful evaluations in the near future. This study has taken valuable steps toward elucidating the complex, defense mechanism responses to biotic and salt stress in plants.

Conclusion. Our work revealed that overexpression of atpA positively regulates plant resistance to $B$. cinerea, as inferred by a balanced ROS, reduced oxidative damage, and increased photosynthesis efficiency compared with WT tobacco plants. Overexpression of the critical atpA gene could modulate greater expression of genes related to plant-pathogen interaction, as well as photosynthetic-related and metabolism genes, during the early stages of $B$. cinerea infection. In addition, atp $A$ overexpression simultaneously improved both salt-stress tolerance and resistance of plants to Cladosporium fulvum, suggesting that it is capable of inducing broad-spectrum defense.

\section{LITERATURE CITED}

Ameline-Torregrosa, C., Wang, B. B., O'Bleness, M. S., Deshpande, S., Zhu, H., Roe, B., Young, N. D., and Cannon, S. B. 2007. Identification and characterization of nucleotide-binding site-leucine-rich repeat genes in the model plant Medicago truncatula. Plant Physiol. 146:5-21.

Arnon, D. I. 1949. Copper enzymes in isolated chloroplasts. Polyphenoloxidase in Beta vulgaris. Plant Physiol. 24:1-15.

Asrar, H., Hussain, T., Hadi, S. M. S., Gul, B., Nielsen, B. L., and Khan, M. A. 2017. Salinity induced changes in light harvesting and carbon assimilating complexes of Desmostachya bipinnata (L.) Staph. Environ. Exp. Bot. 135:86-95.

Audenaert, K., De Meyer, G. B., and Höfte, M. 2002. Abscisic acid determines basal susceptibility of tomato to Botrytis cinerea and suppresses salicylic acid dependent signaling mechanisms. Plant Physiol. 128:491-501.

Buchert, F., Schober, Y., Römpp, A., Richter, M. L., and Forreiter, C. 2012. Reactive oxygen species affect ATP hydrolysis by targeting a highly conserved amino acid cluster in the thylakoid ATP synthase $\gamma$ subunit. Biochim. Biophys. Acta, Bioenerg. 1817:2038-2048.

Bui, T. T., Wright, S. A., Falk, A. B., Vanwalleghem, T., Van Hemelrijck, W., Hertog, M. L., Keulemans, J., and Davey, M. W. 2019. Botrytis cinerea differentially induces post-harvest antioxidant responses in "Braeburn" and "Golden Delicious" apple fruit. J. Sci. Food Agric. 99:5662-5670.

Chen, Y. E., Cui, J. M., Su, Y. Q., Yuan, S., Yuan, M., and Zhang, H. Y. 2015. Influence of stripe rust infection on the photosynthetic characteristics and antioxidant system of susceptible and resistant wheat cultivars at the adult plant stage. Front. Plant Sci. 6:779. doi.org/10.3389/fpls.2015.00779

Conrath, U., Beckers, G. J. M., Langenbach, C. J. G., and Jaskiewicz, M. R. 2015. Priming for enhanced defense. Annu. Rev. Phytopathol. 53:97-119.

Dangl, J. L., Horvath, D. M., and Staskawicz, B. J. 2013. Pivoting the plant immune system from dissection to deployment. Science 341:746-751.

Demidchik, V., Shang, Z., Shin, R., Thompson, E., Rubio, L., Laohavisit, A., Mortimer, J. C., Chivasa, S., Slabas, A. R., Glover, B. J., Schachtman, D. P., Shabala, S. N., and Davies, J. M. 2009. Plant extracellular ATP signalling by plasma membrane NADPH oxidase and $\mathrm{Ca}^{2+}$ channels. Plant J. 58:903-913.

Dodds, P. N., and Rathjen, J. P. 2010. Plant immunity: Towards an integrated view of plant-pathogen interactions. Nat. Rev. Genet. 11:539-548.

Dworak, A., Nykiel, M., Walczak, B., Miazek, A., Szworst-Łupina, D., Zagdańska, B., and Kiełkiewicz, M. 2016. Maize proteomic responses to separate or overlapping soil drought and two-spotted spider mite stresses. Planta 244:939-960.

Gabara, B., Kuźniak, E., Skłodowska, M., Surówka, E., and Miszalski, Z. 2012. Ultrastructural and metabolic modifications at the plant-pathogen interface in Mesembryanthemum crystallinum leaves infected by Botrytis cinerea. Environ. Exp. Bot. 77:33-43.

Göhre, V., Jones, A. M. E., Sklenář, J., Robatzek, S., and Weber, A. P. M. 2012. Molecular crosstalk between PAMP-triggered immunity and photosynthesis. Mol. Plant-Microbe Interact. 25:1083-1092. 
Gong, C., Liu, Y., Liu, S. Y., Cheng, M. Z., Zhang, Y., Wang, R. H., Chen, H. Y., Li, J. F., Chen, X. L., and Wang, A. X. 2017. Analysis of Clonostachys rosea-induced resistance to grey mould disease and identification of the key proteins induced in tomato fruit. Postharvest Biol. Technol. 123:83-93.

Govrin, E. M., and Levine, A. 2000. The hypersensitive response facilitates plant infection by the necrotrophic pathogen Botrytis cinerea. Curr. Biol. 10:751-757.

Hao, L. H., Wang, W. X., Chen, C., Wang, Y. F., Liu, T., Li, X., and Shang, Z. L. 2012. Extracellular ATP promotes stomatal opening of Arabidopsis thaliana through heterotrimeric $\mathrm{G}$ protein $\alpha$ subunit and reactive oxygen species. Mol. Plant 5:852-864.

Horsch, R. B., Fry, J. E., Hoffmann, N. L., Eichholtz, D., Rogers, S. G., and Fraley, R. T. 1985. A simple and general method for transferring genes into plants. Science 227:1229-1231.

Jing, X., Hou, P., Lu, Y., Deng, S., Li, N., Zhao, R., Sun, J., Wang, Y., Han, Y., Lang, T., and Ding, M. 2015. Overexpression of copper/zinc superoxide dismutase from mangrove Kandelia candel in tobacco enhances salinity tolerance by the reduction of reactive oxygen species in chloroplast. Front. Plant Sci. 6:23. doi.org/10.3389/fpls.2015.00023

Jing-Yu, J., Jing, Y., Bo-Wen, Z., Shu-Ren, W., Guo-Cai, Z., and Lian-Nan, L. 2020. Sodium pheophorbide a controls cherry tomato gray mold (Botrytis cinerea) by destroying fungal cell structure and enhancing disease resistancerelated enzyme activities in fruit. Pestic. Biochem. Physiol. 166:104581

Jones, J. D. G., and Dangl, J. L. 2006. The plant immune system. Nature 444: 323-329.

Kanazawa, A., Ostendorf, E., Kohzuma, K., Hoh, D., Strand, D. D., Sato-Cruz, M., Savage, L., Cruz, J. A., Fisher, N., Froehlich, J. E., and Kramer, D. M. 2017. Chloroplast ATP synthase modulation of the thylakoid proton motive force: Implications for photosystem I and photosystem II photoprotection. Front. Plant Sci. 8:719.

Kangasjärvi, S., Tikkanen, M., Durian, G., and Aro, E. M. 2014. Photosynthetic light reactions-an adjustable hub in basic production and plant immunity signaling. Plant Physiol. Biochem. 81:128-134.

Kim, D., Pertea, G., Trapnell, C., Pimentel, H., Kelley, R., and Salzberg, S. L. 2013. Tophat2: Accurate alignment of transcriptomes in the presence of insertions, deletions and gene fusions. Genome Biol. 14:R36.

Kramer, D. M., Avenson, T. J., and Edwards, G. E. 2004. Dynamic flexibility in the light reactions of photosynthesis governed by both electron and proton transfer reactions. Trends Plant Sci. 9:349-357.

Landoni, M., De Francesco, A., Bellatti, S., Delledonne, M., Ferrarini, A., Venturini, L., Pilu, R., Bononi, M., and Tonelli, C. 2013. A mutation in the FZL gene of Arabidopsis causing alteration in chloroplast morphology results in a lesion mimic phenotype. J. Exp. Bot. 14:14.

Leyva, J. A., Bianchet, M. A., and Amzel, L. M. 2003. Understanding ATP synthesis: Structure and mechanism of the F1-ATPase. Mol. Membr. Biol. 20:27-33.

Li, R., Sheng, J., and Shen, L. 2020. Nitric oxide plays an important role in $\beta$-aminobutyric acid-induced resistance to Botrytis cinerea in tomato plants. Plant Pathol. J. 36:121-132.

Li, Y., Zhang, Y., Wang, Q. X., Wang, T. T., Cao, X. L., Zhao, Z. X., Zhao, S. L., Xu, Y. J., Xiao, Z. Y., Li, J. L., and Fan, J. 2018. RESISTANCE TO POWDERY MILDEW8.1 boosts pattern-triggered immunity against multiple pathogens in Arabidopsis and rice. Plant Biotechnol. J. 16:428-441.

Lindenthal, M., Steiner, U., Dehne, H. W., and Oerke, E. C. 2005. Effect of downy mildew development on transpiration of cucumber leaves visualized by digital infrared thermography. Phytopathology 95:233-240.

Liu, X., Lin, F., Wang, L., and Pan, Q. 2007. The in silico map-based cloning of Pi36, a rice coiled-coil nucleotide-binding site leucine-rich repeat gene that confers race-specific resistance to the blast fungus. Genetics 176: 2541-2549.

Liu, X., Ma, F., Zhu, H., Ma, X., Guo, J., Wan, X., Wang, L., Wang, H., and Wang, Y. 2017. Effects of magnetized water treatment on growth characteristics and ion absorption, transportation and distribution in Populus $\times$ euramericana 'Neva' under $\mathrm{NaCl}$ stress. Can. J. For. Res. 47:828-838.

Martinez-Medina, A., Flors, V., Heil, M., Mauch-Mani, B., Pieterse, C. M., Pozo, M. J., Ton, J., van Dam, N. M., and Conrath, U. 2016. Recognizing plant defense priming. Trends Plant Sci. 21:818-822.

Mengiste, T. 2012. Plant immunity to necrotrophs. Annu. Rev. Phytopathol. 50:267-294

Mouekouba, L. D., Zhang, L., Guan, X., Chen, X., Chen, H., Zhang, J., Zhang, J., Li, J., Yang, Y., and Wang, A. 2014. Analysis of Clonostachys rosea induced resistance to tomato gray mold disease in tomato leaves. PLoS One 9e102690.

Munis, M. F. H., Tu, L., Deng, F., Tan, J., Xu, L., Xu, S., Long, L., and Zhang, X. 2010. A thaumatin-like protein gene involved in cotton fiber secondary cell wall development enhances resistance against Verticillium dahliae and other stresses in transgenic tobacco. Biochem. Biophys. Res. Commun. 393:38-44

Murray, M. G., and Thompson, W. F. 1980. Rapid isolation of high molecularweight plant DNA. Nucleic Acids Res. 8:4321-4326.

Nemchinov, L. G., Lana, S., and Sergey, S. 2008. Calcium efflux as a component of the hypersensitive response of Nicotiana benthamiana to Pseudomonas syringae. Plant Cell Physiol. 49:40-46.

Patel, T. K., Krasnyanski, S. F., Allen, G. C., Louws, F. J., Panthee, D. R., and Williamson, J. D. 2015. Tomato plants overexpressing a celery mannitol dehydrogenase (MTD) have decreased susceptibility to Botrytis cinerea. Am. J. Plant Sci. 6:1116-1125.

Seifi, H. S., Curvers, K., De Vleesschauwer, D., Delaere, I., Aziz, A., and Höfte, M. 2013. Concurrent overactivation of the cytosolic glutamine synthetase and the GABA shunt in the ABA-deficient sitiens mutant of tomato leads to resistance against Botrytis cinerea. New Phytol. 199: $490-504$

Song, C. J., Steinebrunner, I., Wang, X., Stout, S. C., and Roux, S. J. 2006. Extracellular ATP induces the accumulation of superoxide via NADPH oxidases in Arabidopsis. Plant Physiol. 140:1222-1232.

Song, Y., Chen, Q., Ci, D., Shao, X., and Zhang, D. 2014. Effects of high temperature on photosynthesis and related gene expression in poplar. BMC Plant Biol. 14:111.

Takagi, D., Amako, K., Hashiguchi, M., Fukaki, H., Ishizaki, K., Goh, T., Fukao, Y., Sano, R., Kurata, T., Demura, T., Sawa, S., and Miyake, C. 2017. Chloroplastic ATP synthase builds up a proton motive force preventing production of reactive oxygen species in photosystem I. Plant J. 91: 306-324.

Tameling, W. I. L., Elzinga, S. D. J., Darmin, P. S., Vossen, J. H., Takken, F. L. W., and Cornelissen, B. J. C. 2002. The tomato R gene products I-2 and $\mathrm{Mi}-1$ are functional ATP binding proteins with ATPase activity. Plant Cell 14:2929-2939.

Tanaka, K., Choi, J., Cao, Y., and Stacey, G. 2014. Extracellular ATP acts as a damage-associated molecular pattern (DAMP) signal in plants. Front. Plant Sci. 5:446.

Verhagen, B. W., Trotel-Aziz, P., Couderchet, M., Hofte, M., and Aziz, A. 2009. Pseudomonas spp.-induced systemic resistance to Botrytis cinerea is associated with induction and priming of defence responses in grapevine. J. Exp. Bot. 61:249-260.

von Ballmoos, C., Wiedenmann, A., and Dimroth, P. 2009. Essentials for ATP synthesis by $\mathrm{F}_{1} \mathrm{~F}_{0}$ ATP synthases. Annu. Rev. Biochem. 78:649-672.

Wang, G., Cai, G., Kong, F., Deng, Y., Ma, N., and Meng, Q. 2014. Overexpression of tomato chloroplast-targeted DnaJ protein enhances tolerance to drought stress and resistance to Pseudomonas solanacearum in transgenic tobacco. Plant Physiol. Biochem. 82:95-104.

Wang, Q., Chen, X., Chai, F., Xue, D., Zheng, W., Shi, Y., and Wang, A. 2019. The involvement of jasmonic acid, ethylene and salicylic acid in the signaling pathway of Clonostachys rosea-induced resistance to gray mold disease in tomato. Phytopathology 109:1102-1114.

Wang, R. 2013. Clonostachys rosea induced non-host resistance to gray mould disease in tomato. Master's dissertation (in Chinese). Northeast Agricultural University, Harbin, China.

Wei, T., Wang, Y., Xie, Z., Guo, D., Chen, C., Fan, Q., Deng, X., and Liu, J. H. 2019. Enhanced ROS scavenging and sugar accumulation contribute to drought tolerance of naturally occurring autotetraploids in Poncirus trifoliata. Plant Biotechnol. J. 17:1394-1407.

Xu, Q., Tang, C., Wang, X., Sun, S., Zhao, J., Kang, Z., and Wang, X. 2019. An effector protein of the wheat stripe rust fungus targets chloroplasts and suppresses chloroplast function. Nat. Commun. 10:5571.

Xue, C., Liu, Z., Dai, L., Bu, J., Liu, M., Zhao, Z., Jiang, Z., Gao, W., and Zhao, J. 2018. Changing host photosynthetic, carbohydrate and energy metabolisms play important roles in phytoplasma infection. Phytopathology 108:1067-1077.

Xue, D. Q., Chen, X. L., Zhang, H., Chai, X. F., Jiang, J. B., Xu, X. Y., and Li, J. F. 2017. Transcriptome analysis of the $C f$-12-mediated resistance response to Cladosporium fulvum in tomato. Front. Plant Sci. 7:2012.

Yang, Q., Huai, B., Lu, Y., Cai, K., Guo, J., Zhu, X., Kang, Z., and Guo, J. 2020. A stripe rust effector Pst 18363 targets and stabilises TaNUDX23 that promotes stripe rust disease. New Phytol. 225:880-895.

Yang, S., Li, X., Chen, W., Liu, T., and Luo, G. P. 2016. Wheat resistance to fusarium head blight is associated with changes in photosynthetic parameters. Plant Dis. 100:847-852.

Zhao, Y., Lim, J., Xu, J., Yu, J., and Zheng, W. 2020. Nitric oxide as a developmental and metabolic signal in filamentous fungi. Mol. Microbiol. 113:872-882. doi.org/10.1111/mmi.14465 